УДК 004.03

Архангельская Екатерина Владиславовна

кандидат физико-математических наук, доцент, доцент

Саратовская государственная юридическая академия, г. Саратов, Россия

ORCID ID 0000-0003-4108-9876

katysar@rambler.ru

\title{
ВОЗМОЖНОСТИ ИСПОЛЬЗОВАНИЯ МІСRОSОFТ ЕХСЕL ДЛЯ РАЗРАБОТКИ ПРОГРАММНЫХ СРЕДСТВ РЕШЕНИЯ ЗАДАЧ ДИНАМИЧЕСКОГО ПРОГРАММИРОВАНИЯ
}

\begin{abstract}
Аннотация. В статье описывается процесс создания и разработки электронного пособия для изучения метода динамического программирования. Данный метод используется для решения некоторых задач оптимизации и изучается в рамках таких дисциплин, как «Теория принятия решения» и «Методы оптимальных решений». В статье освещены возможности использования разработанного пособия для формирования условия и решения практической задачи об оптимальном распределении ресурсов между четырьмя предприятиями, определении максимального дохода и размеров средств, вкладываемых в каждое предприятие. Показано, каким образом можно обеспечить уникальность вариантов рассматриваемой задачи на каждом компьютере. Продемонстрирована реализация автоматической проверки правильности ответа, введенного пользователем, и показа расчетов, получаемых на разных этапах решения задачи, в мультимедийном режиме. При постановке задачи о распределении ресурсов и проведении расчетов методом динамического программирования исходные данные и результаты расчетов записываются в виде таблиц. По этой причине в качестве программного продукта для разработки пособия был выбран широко распространенный табличный процессор Microsoft Excel. Для функционирования программной разработки применяется программирование в среде VBA Excel. Формирование условия задачи осуществляется с помощью программного кода, выполняемого при открытии файла электронного пособия. В случае если у пользователя возникают затруднения при решении задачи, он может просмотреть процесс решения в мультимедийном режиме. В статье излагается, как можно организовать показ решения при нажатии кнопок, расположенных на листе Excel. Электронное пособие представляет собой цельный программный продукт, успешно используемый на практических занятиях в компьютерных классах. Пособие может использоваться для самостоятельного и дистанционного обучения.
\end{abstract}

Ключевые слова: электронные учебные ресурсы; электронное пособие; динамическое программирование; задача об оптимальном распределении ресурсов; автоматическая проверка решения; мультимедийность.

\section{1. ВВЕДЕНИЕ}

Постановка проблемы. Изучение методов оптимизации и методов принятия решений входят в программу базовых дисциплин и дисциплин по выбору студентов различных специальностей как естественнонаучных направлений, так и экономических, юридических и др. В рамках специального и высшего образования большое внимание уделяется методам линейного программирования, а также методам целочисленного и динамического программирования. Задачи линейной оптимизации достаточно широко представлены в учебной литературе и на учебных информационных порталах. На различных сайтах можно найти большое количество формулировок практических задач, on-line-калькуляторы, предоставляющие подробное решение. Находить решение задач линейного программирования можно также с помощью общедоступных прикладных программ, например, используя табличный процесcop Microsoft Excel. Ha 
изучение методов динамического программирования выделяется гораздо меньшее количество часов, поэтому они не так широко представлены в учебниках, и пособиях, и в информационных сетях. Для изучения данного раздела оптимизации необходимы разработка и использование методических материалов, учитывающих особенности специальности и учебного заведения.

C внедрением информационных технологий в учебный процесс все чаще на занятиях используются электронные учебники и электронные учебные пособия, разрабатываемые самими преподавателями. Электронные пособия особенно актуальны, когда занятия проводятся в компьютерном классе и каждый обучаемый может выполнять задания на компьютере. При создании электронных учебно-методических материалов для изучения методов динамического программирования нужно реализовать возможность формирования условия конкретной задачи, проверки правильности решения и отображение основных этапов решения задачи.

Анализ последних исследований и публикаций. При современном этапе развития информационных технологий процесс обучения в учебных заведениях высшего профессионального образования невозможно представить без их использования. Информационные технологии используются как средства проверки знаний (всевозможные тестирующие программы), как средства коммуникации преподавателя и студента (дистанционные обучающие программы, вебинары и т.п.) Несмотря на наличие и большой выбор компьютерных программ, учебных сайтов, электронных учебников, потребность в них с каждым днем возрастает. Меняются программы дисциплин, учебные планы, появляются новые дисциплины - все это приводит к необходимости создания новых компьютерных ресурсов обучения.

Использование компьютерных технологий в обучении студентов различных специальностей широко обсуждается в научных статьях. Большое внимание уделяется формированию компетенций с учетом применения в изучении дисциплин информационных ресурсов. Авторами по различным критериям оцениваются программно-имитационные комплексы для формирования компетентностей студентов технических специальностей [1]. Проводятся исследования особенностей формирования компетентностей экономистов в процессе получения образовательных услуг в высших учебных заведениях, в том числе с использованием информационнокоммуникационных технологий [2]. Раскрываются особенности применения информационных систем управления проектами, приводится обоснование применения информационных систем и технологий в подготовке проект-менеджеров [3]. В научных работах доказывается, что применение информационных технологий в обучении, необходимо, т.к. современные требования к подготовке специалистов заставляют преподавателей совершенствовать формы, методы обучения так, чтобы формировать у студентов гибкие знания, умения, способность быстро адаптироваться к стремительным темпам научно-технического прогресса и новым информационным технологиям, в том числе при профессиональной подготовке будущих менеджеровэкономистов [4].

Преподаватели разрабатывают свои информационные ресурсы с учетом специфики специальности, специфики вуза, а также с учетом собственных возможностей - доступа к программным средствам, умением программировать в различных средах и т.п.

Преподавателями активно разрабатываются электронные учебные материалы, доступ к которым можно получить без ограничений в сети Интернет или на портале вуза. Электронные учебные пособия, которые создаются по различным дисциплинам [5], могут содержать как теоретические материалы, так и практические задания и 
представляют собой файлы, созданные в различных программах, например, файлы презентаций, текстовые файлы, программные файлы и т.п.

Достаточно широко для разработки электронных образовательных ресурсов используется среда СКМ Maple, которая, по мнению авторов, предоставляет возможность разработки учебной среды с более мощным инструментарием по сравнению с текстовым редактором [6]. В частности, авторами обоснована необходимость использования данной среды для обновления учебных задач линейного программирования с целью освобождения студентов от громоздких однотипных арифметических вычислений и записей, что ведет к более глубокому пониманию ключевых идей, которые положены в основу используемых алгоритмов [7].

Эффективность применения различных электронных пособий также широко обсуждается в научных публикациях [8]. Результаты экспериментов авторов показывают эффективность внедрения методов, в том числе с использованием системы компьютерной алгебры Maple-тренажеров для повышения мотивации участников образовательного процесса, установления обратной связи с каждым студентом на определенных этапах обучения [9]. Многими авторами используются мультимедийные эффекты для более наглядного и качественного представления материала [10], [11]. Важным при выборе программного продукта для создания электронных учебников является распространенность и доступность программных средств, предпочтение отдается продуктам свободно распространяемого программного обеспечения [12], [13].

Учитывая вышеизложенное, целью статьи является описание процесса разработки и создания электронного учебного пособия по изучению методов динамического программирования и их применения для решения задачи оптимизации.

\section{2. РЕЗУЛЬТАТЫ ИССЛЕДОВАНИЯ}

\section{1. Концепции разработки электронного пособия}

Электронное пособие разрабатывалось с использованием широко применяемой программы Microsoft Excel. При анализе решения практических задач в пособии используются мультимедийные эффекты.

Пособие, процесс создания и использования которого описывается в статье, представляет собой файл, созданный в табличном процессоре Microsoft Excel. Выбор данного программного продукта обусловлен, в первую очередь, его широкой распространенностью, а также наличием встроенных математических функций и возможностью применять элементы программирования в среде Visual Basic Application (VBA). Одной из основных задач, с которых начинается изучение метода динамического программирования, является задача об оптимальном распределении средств. Назначение электронного учебного пособия заключается, прежде всего, в формировании условия задачи о распределении средств. Пользователю предлагается решить практическую задачу, представленную в файле пособия, и ввести ответ. После чего в пособии происходит автоматическая проверка правильности введенного ответа. Если у пользователя возникают трудности при решении задачи, в пособии можно просмотреть результаты расчетов на всех этапах решения и формирование оптимального решения поставленной задачи.

Функциональность всех компонент электронного пособия - формирование условия задачи, проверка правильности решения и показ решения - разработана с помощью программирования в среде VBA или с использованием встроенных функций табличного процесcopa Microsoft Excel. Числовые значения в условии практических задач формируются случайным образом, таким образом, обеспечивается создание 
различных вариантов заданий на каждом компьютере. При отображении показа решения для наглядности используются мультимедийные эффекты. Возможность просмотра этапов решения позволяет использовать разработанное электронное пособие для самостоятельного и дистанционного обучения. Ниже представлены этапы создания всех компонент описываемой компьютерной разработки.

\section{2. Задача об оптимальном распределении средств}

При изучении дисциплин «Теория принятия решения» и «Методы оптимальных решений» рассматриваются задачи, процесс решения которых разбивается на шаги. Оптимальное решение принимается с учетом выбора наилучшего значения на каждом шаге. К таким задачам относятся задачи динамического программирования. В «Теории принятия решений» решаются задачи, создающие систему управленческих действий, обеспечивающую реализацию сложной системной деятельности лиц, принимающих решения, на научной основе, а именно задача об определении оптимальных сроков замены оборудования, задача о распределении средств между объектами, задача о составлении оптимального расписания выполнения работ. В «Методах оптимальных решений» динамическое программирование применяется к решению задачи об определении кратчайшего пути в сети.

Задача о распределении средств, для решения которой применяется метод динамического программирования, формулируется следующим образом.

Начальную сумму средств $\xi_{0}$ планируется распределить между $n$ предприятиями $\Pi_{1}, \Pi_{2}, \ldots, \Pi_{n}$. Выделенные средства предприятию $\Pi_{k}$ в размере $x_{k}$ приносят доход $f_{k}\left(x_{k}\right) \quad(k=1,2, \ldots, n)$. Общий доход равен сумме доходов, полученных от распределения всех средств по всем предприятиям. Математическая модель задачи имеет вид:

доход от распределения средств выражается целевой функцией

$$
Z=\sum_{k=1}^{n} f_{k}\left(x_{k}\right),
$$

переменные $x_{k}$ должны удовлетворять условиям

$$
x_{1}+x_{2}+\ldots+x_{n}=\xi_{0}, x_{k} \geq 0,(k=1,2, \ldots, n) .
$$

Требуется определить такие значения переменных $x_{1}, x_{2}, \ldots, x_{n}$, которые удовлетворяют ограничениям (2) и при которых целевая функция (1) принимает максимальное значение.

Решение поставленной задачи методом динамического программирования осуществляется в два этапа. Первый этап носит название условной оптимизации, второй - безусловной оптимизации. Этап условной оптимизации представляет собой $n$ шаговый процесс, где номером $k$-го шага является номер предприятия, которому выделяются средства. На каждом шаге система характеризуется величиной $\xi_{k-1}-$ остатком средств после распределения на предшествующих шагах. Данные величины связаны с переменными $x_{k}$ следующим уравнением состояния системы $\xi_{k}=\xi_{k-1}-x_{k}$. Вычисления начинаются с последнего $n$-го шага, на котором выделяются средства последнему предприятию, они определяются по формуле:

$$
Z_{n}^{*}\left(\xi_{n-1}\right)=\max _{0 \leq x_{n} \leq \xi_{n-1}}\left\{f_{n}\left(x_{n}\right)\right\} .
$$

На всех остальных шагах средства, выделяемые предприятию $\Pi_{k}$ находятся из уравнения: 


$$
Z_{k}^{*}\left(\xi_{k-1}\right)=\max _{0 \leq x_{k} \leq \xi_{k-1}}\left\{f_{k}\left(x_{k}\right)+Z_{k+1}^{*}\left(\xi_{k}\right)\right\} .
$$

В результате условной оптимизации после последовательного прохождения всех шагов от последнего $n$-го шага к первому получаем на каждом шаге значения условных максимальных доходов $Z_{n}^{*}\left(\xi_{n-1}\right), \quad Z_{n-1}^{*}\left(\xi_{n-2}\right), \quad \ldots, \quad Z_{2}^{*}\left(\xi_{1}\right), \quad Z_{1}^{*}\left(\xi_{0}\right)$ и условные оптимальные значения переменных $x_{n}^{*}\left(\xi_{n-1}\right), x_{n-1}^{*}\left(\xi_{n-2}\right), \ldots, x_{2}^{*}\left(\xi_{1}\right), x_{1}^{*}\left(\xi_{0}\right)$. Затем переходим к безусловной оптимизации. Зная начальное значение $\xi_{0}$, определяется максимальный доход $Z_{1}^{*}\left(\xi_{0}\right)$ и количество средств $x_{1}^{*}\left(\xi_{0}\right)$, выделяемых 1-му предприятию. Далее определяем остаток средств из уравнения состояния системы $\xi_{1}^{*}=\xi_{0}-x_{1}^{*}$, по данной величине находим $x_{2}^{*}\left(\xi_{1}^{*}\right)$ и т.д. переходим от первого шага к последнему, определяя искомые оптимальные значения переменных.

\section{3. Формирование условия практических задач в файле пособия}

Файл разработанного электронного пособия для изучения применения метода динамического программирования к решению задачи об оптимальном распределении средств является книгой Excel с поддержкой макросов. После открытия файла на листе формируется условие задачи, которую пользователю предлагается решить и ввести ответ в указанные ячейки листа файла Excel. На практике целесообразно решать задачи, в которых необходимо распределить указанную сумму средств между четырьмя предприятиями $\Pi_{1}, \Pi_{2}, \Pi_{3}, \Pi_{4}$. Приведем пример конкретной формулировки задачи: необходимо распределить средства в размере $\xi_{0}=500$ ден. ед. между четырьмя предприятиями так, чтобы общий доход от вложения был максимальным; средства выделяются в размерах, кратных 100; размеры дохода в зависимости от вложенных средств каждого предприятия заданы в таблице. Как видно из формулировки, начальными исходными числовыми данными являются значение $\xi_{0}$, размеры переменных $x_{k}$ и таблица доходов $f_{k}\left(x_{k}\right)$. В данной задаче переменные могут принимать шесть значений - 0, 100, 200, 300, 400, 500. В пособии также формируются условия задач со следующими параметрами: $\xi_{0}=250$, при этом средства выделяются в размерах, кратных 50, и $\xi_{0}=1000$, при этом средства выделяются в размерах, кратных 200. Во всех задачах средства вкладываются в четыре предприятия, и переменные $x_{k}$ могут принимать шесть значений. Очевидно, что этапы решения всех представленных в пособии задач совпадают, также совпадают все вычислительные и алгоритмические процессы, необходимые для реализации представления и решения задач с помощью программирования. Однако такой подход обеспечивает различие формулировок задач и проявление большей самостоятельности студентами при их решении и работе с пособием.

Формирование условий задач обеспечивается работой программного кода, созданного в среде программирования VBA Excel. Формирование происходит при открытии файла электронного пособия, для этого соответствующий программный код записан внутри процедуры Workbook_Open() объекта Workbook. При открытии файла на листе Excel отображается один из трех вариантов формулировка задачи об оптимальном распределении ресурсов, описанных выше (рис. 1). 


\begin{tabular}{|c|c|c|c|c|c|c|}
\hline & A & B & $\mathrm{C}$ & D & $E$ & $\mathrm{~F}$ \\
\hline 1 & \multicolumn{6}{|c|}{$\begin{array}{l}\text { Распределить средства в размере } \xi_{0}=250 \\
\text { ден. ед. между четырьмя предприятиями } \\
\text { так, чтобы общий доход от вложения был } \\
\text { максимальным; средства выделяются в } \\
\text { размерах, кратных 50; размеры дохода в } \\
\text { зависимости от вложенных средств } \\
\text { каждого предприятия заданы в таблице }\end{array}$} \\
\hline 2 & $x \bigvee \Pi$ & $f_{1}(x)$ & $f_{2}(x)$ & $f_{3}(x)$ & $f_{4}(x)$ & \\
\hline 3 & 0 & 0 & 0 & 0 & 0 & \\
\hline 4 & 50 & 7 & 12 & 8 & 8 & \\
\hline 5 & 100 & 14 & 13 & 17 & 15 & \\
\hline 6 & 150 & 24 & 15 & 21 & 27 & \\
\hline 7 & 200 & 37 & 39 & 37 & 37 & \\
\hline 8 & 250 & 41 & 43 & 43 & 40 & \\
\hline
\end{tabular}

\begin{tabular}{|c|c|c|c|c|c|c|}
\hline & $\mathrm{A}$ & $\mathrm{B}$ & $\mathrm{C}$ & $\mathrm{D}$ & $\mathrm{E}$ & $\mathrm{F}$ \\
\hline 1 & \multicolumn{6}{|c|}{ Распределить средства в размере $\xi_{0}=500$} \\
ден. ед. между четырьмя предприятияи \\
так, чтобы общий доход от вложения был \\
максимальным; средства выделяются в \\
размерах, кратных 100; размеры дохода в \\
зависимости от вложенных средств каждого \\
предприятия заданы в таблице
\end{tabular}

Рис. 1. Варианты практических задач в файле электронного пособия

Один из трех вариантов формулировки задачи определяется случайным образом, для этого в программе запускается генератор случайных чисел командой Randomize и задается параметр $\mathrm{k}=\operatorname{Int}((3-1+1) * \mathrm{Rnd}+1)$, выражение для которого определяет случайное число от 1 до 3. Программный код, отображающий условие задачи в ячейке A1 и размеры вкладываемых средств в ячейках A4:A8, представлен ниже:

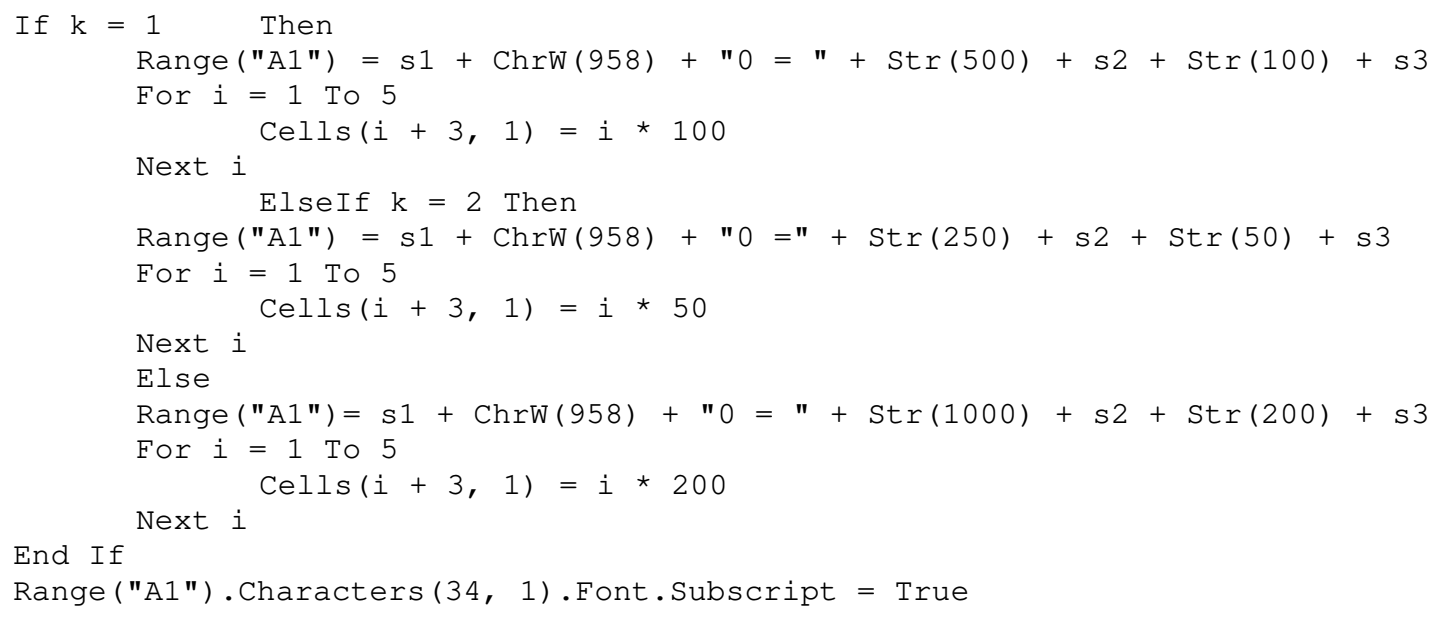

В данном коде значениями строковых переменных s1, s2, s3 являются фиксированные текстовые фрагменты условия задачи. Оператор $\mathrm{ChrW}(958)$ добавляет в строку символ $\xi$ по его коду, последний оператор делает подстрочным символ 0.

Затем в условии задачи формируется таблица значений $f_{k}\left(x_{k}\right)$ (рис. 1$)$. Основной целью разработки является изучение метода динамического программирования, поэтому нет необходимости выполнять вычисления с большими числами, поэтому значения таблицы являются случайными числами от 5 до 50. Функции дохода $f_{k}$, исходя из практического смысла задачи, монотонно возрастают, поэтому задавать числовые значения нужно так, чтобы большему значению $x_{k}$ соответствовало большее значение $f_{k}\left(x_{k}\right)$. Значения в ячейках $\mathrm{B} 4: \mathrm{E} 4$, соответствующие меньшему значению $f_{k}\left(x_{k}\right)$, являются случайными числами от 5 до 15 и в программном коде задаются с помощью выражения $\operatorname{Int}((15-5+1) * \operatorname{Rnd}+5)$. Числа в ячейках B5:E5 являются 
случайными числами в диапазоне от значения $f_{k}\left(x_{1}\right)$ до 25 , т.е., например, число в ячейке В5 может принимать значение в диапазоне между числом из ячейки В4 и числом 25. Аналогично задаются числа в ячейках B6:E6, B7:E7 и В8:E8, они являются случайными числами в диапазоне от числа из ячейки, расположенной в предыдущей строке и этом же столбце, до 50. Значения ячеек В4:Е8 формируются в результате выполнения следующего программного кода:

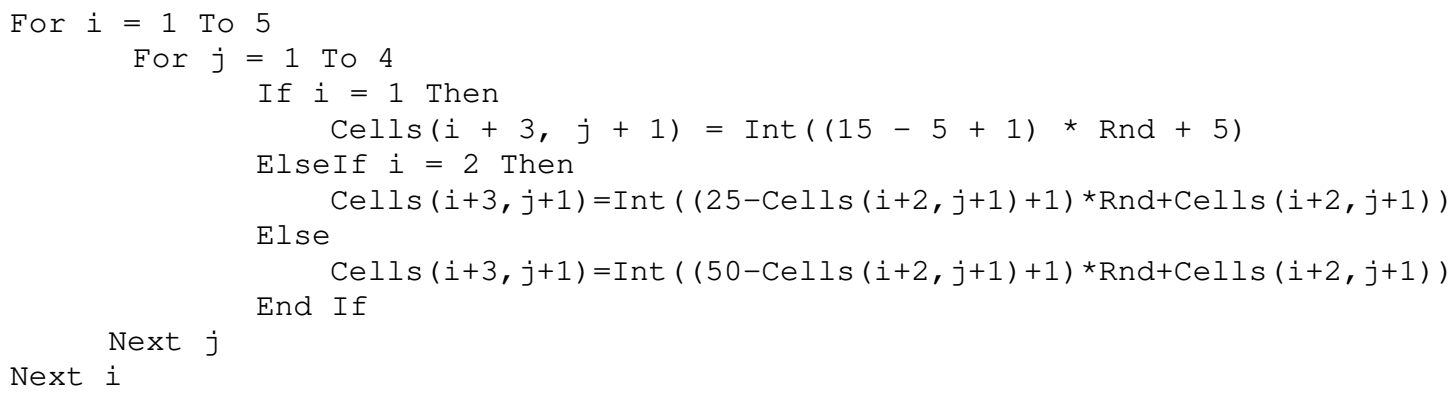

\section{4. Организация автоматической проверки решения}

После открытия файла пособия пользователю нужно решить поставленную задачу и ввести ответ в указанные ячейки F11:F15 (рис. 2).

\begin{tabular}{|c|c|c|c|c|c|c|c|}
\hline & A & B & $\mathrm{C}$ & $\mathrm{D}$ & $\mathrm{E}$ & $\mathrm{F}$ & $\mathrm{G}$ \\
\hline & \multicolumn{6}{|l|}{..... } & \\
\hline 10 & \multicolumn{4}{|c|}{ Введите найденное решение: } & & & \\
\hline 11 & \multicolumn{4}{|c|}{ Максимальный получаемый доход } & $Z^{*}=$ & & \\
\hline 12 & \multicolumn{4}{|c|}{ Средства, вкладываемые в 1-е предприятие } & $\mathrm{X}_{1}^{*}=$ & & \\
\hline 13 & \multicolumn{4}{|c|}{ Средства, вкладываемые во 2-е предприятие } & $\mathrm{x} *_{2}=$ & & \\
\hline 14 & \multicolumn{4}{|c|}{ Средства, вкладываемые в 3-е предприятие } & $\mathrm{x} *_{3}=$ & & \\
\hline 15 & \multicolumn{4}{|c|}{ Средства, вкладываемые в 4-е предприятие } & $\mathrm{X} *{ }_{4}=$ & & \\
\hline
\end{tabular}

Рис. 2. Ячейки для ввода найденньх оптимальных значений

Для организации автоматической проверки правильности найденного пользователем решения необходимо сравнить значения, введенные в ячейки F11:F15, с оптимальными значениями переменных и целевой функции, являющихся решением задачи. В пособии решение задачи определяется программным способом по алгоритму метода динамического программирования, и найденные значения переменных записываются в ячейки файла пособия, скрытые от пользователя. Все результаты вычислений на этапе условной оптимизации, согласно классической записи метода динамического программирования, представлены в виде таблицы. Этим определяется выбор в качестве программного продукта для создания электронного пособия табличного процессора, т.к. нет необходимости создавать массивы для хранения переменных, их значения можно записывать в ячейки таблицы.

Результаты расчетов условной оптимизации записываются в ячейки A22:I27. B ячейки A22:A27 записываются значения, которые может принимать управляющая переменная $\xi_{k-1}$, т.е. остаток средств от вложения на каждом шаге, это значения ячеек A3:А8 из условия задачи (рис. 1). В файле пособия это осуществляется с помощью формул Excel, так, например, в ячейку A22 введена формула “=A3". На 4-ом шаге при вложении средств в предприятие П 4 условные оптимальные значения определяются по 
формуле (3), т.е. ищется максимальное значение из одного возможного, на этом шаге величины $Z_{4}^{*}\left(\xi_{3}\right)$ совпадают с $f_{4}(x)$, поэтому в ячейки В22:В27 записываются данные из ячеек E3:E8, а в ячейки C22:C27 данные из ячеек A3:A8 (рис. 1). Расчеты на всех остальных шагах и запись результатов в ячейки D22:I27 осуществляются с помощью программного кода, представленного ниже:

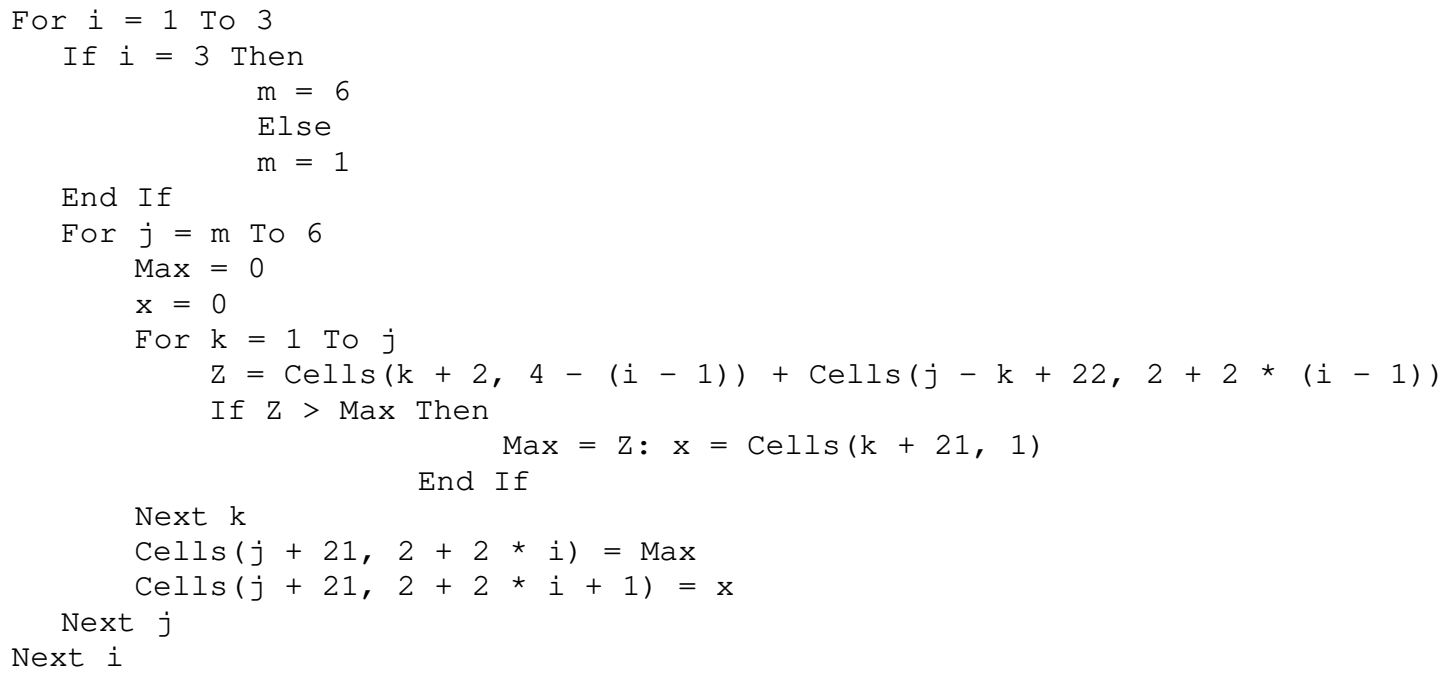

Таким образом, оптимальное значение целевой функции $Z$ * помещается в ячейку H27, оптимальное значение переменной $x_{1}^{*}$ - в ячейку I27. Напомним, что все ячейки с расчетами скрыты от пользователя. Далее в программном коде определяются значения всех остальных переменных, согласно формулам безусловной оптимизации, эти значения записываются в ячейки АА22, АА23, АА24. Сравнить значения, введенные пользователем в качестве ответа, с решением задачи можно с помощью логической функции ЕСЛИ, и ввести нужные функции, например, в ячейки G11:G15. В файле пособия в ячейку G11 введена функция ЕСЛИ(F11="";"";ЕСЛИ(F11=H27;"Верно!";"Не верно!!!")), которая отображает в ячейке слово «Верно!», если в ячейку F11 введено правильное значение максимального дохода. В ячейки G12:G15 введены аналогичные функции сравнения значений двух ячеек. Для большей наглядности к ячейкам G11:G15 применено условное форматирование, в результате которого значение «Не верно!!!» выделяется шрифтом красного цвета. Таким образом, после ввода найденного решения можно сразу определить, является ли оно правильным.

\section{5. Мультимедийный показ этапов решения задачи}

В случае, если у пользователя возникают трудности при решении задачи, в файле пособия предусмотрен показ результатов вычислений, которые необходимо выполнить на этапе условной оптимизации, и оптимальных значений, получаемых в процессе безусловной оптимизации.

\subsection{1. Последовательное отображение результатов расчетов условной оптимизации}

Результаты расчетов, как было сказано выше, записываются в ячейки A22:I27 в результате выполнения программного кода, выполняемого при открытии файла пособия. В указанных ячейках цвет шрифта задан белым и строки с 22:27 скрыты от пользователя. Показ результатов заключается в отображении содержимого указанных ячеек. В файле пособия размещена кнопка с текстом «Показать результаты расчетов 
условной оптимизации», при нажатии которой пользователь сможет увидеть соответствующие числа. Для наглядности, т.к. этап условной оптимизации выполняется по шагам, то в пособии осуществляется последовательное отображение результатов каждого шага. Для этого в программном коде используется цикл задержки времени [14]. Код программы, выполняемой при щелчке кнопки для показа результатов расчета, представлен ниже:

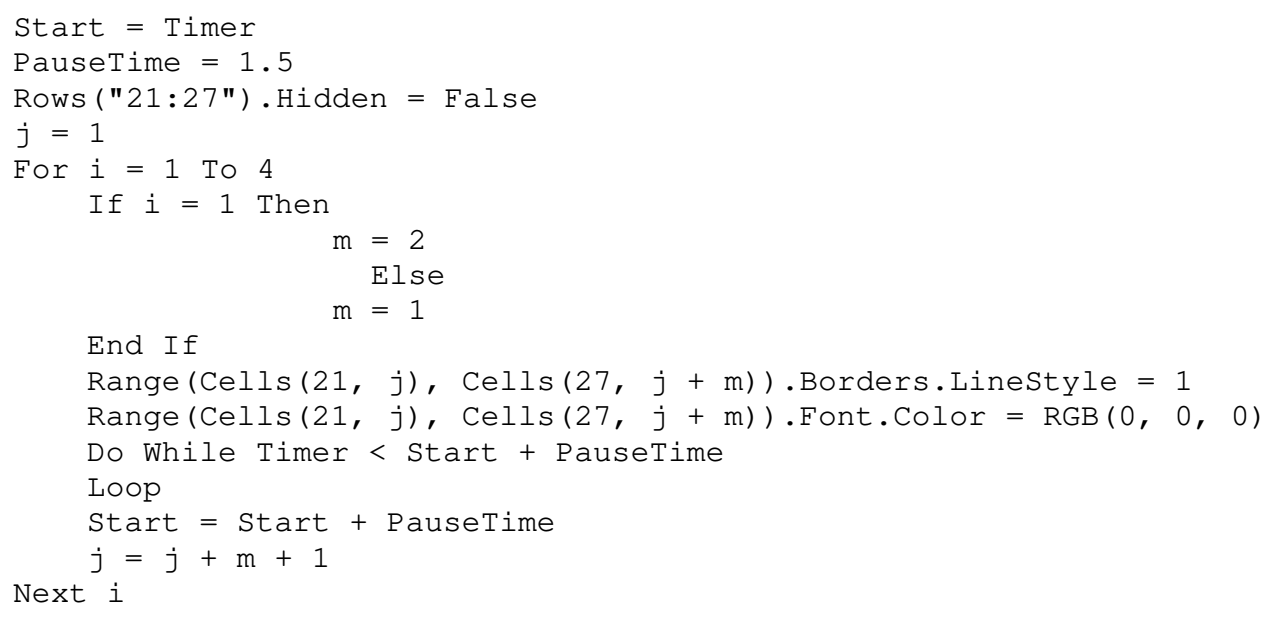

В данном коде сначала ячейкам A21:C27, в которых записаны результаты вычислений 4-го шага условной оптимизации, задается цвет шрифта черный и граница вокруг ячеек, тем самым их содержимое становится видимым для пользователя. Затем через временной интервал, заданный 1,5с (значение переменной PauseTime), то же самое происходит с ячейками D21:E27, в которых записаны результаты вычислений 3го шага, и т.д. Таким образом, пользователь на экране видит последовательное отображение результатов каждого шага (рис. 3).

\begin{tabular}{|c|c|c|c|c|}
\hline & A & B & C & D \\
\hline & $\cdots$ & & & \\
\hline 21 & $\xi_{\mathrm{k}-1}$ & $\mathrm{Z}^{*}{ }_{4}\left(\xi_{3}\right)$ & $\mathrm{X}_{4}\left(\xi_{3}\right)$ & \\
\hline 22 & 0 & 0 & 0 & \\
\hline 23 & 100 & 15 & 100 & \\
\hline 24 & 200 & 21 & 200 & \\
\hline 25 & 300 & 44 & 300 & \\
\hline 26 & 400 & 44 & 400 & \\
\hline 27 & 500 & 50 & 500 & \\
\hline
\end{tabular}

\begin{tabular}{|c|c|c|c|c|c|c|}
\hline & $\mathrm{A}$ & $\mathrm{B}$ & $\mathrm{C}$ & $\mathrm{D}$ & $\mathrm{E}$ & $\mathrm{F}$ \\
\hline & $\cdots$ \\
\hline 21 & $\xi_{\mathrm{k}-1}$ & $\mathrm{Z} *_{4}\left(\xi_{3}\right)$ & $\mathrm{X} *_{4}\left(\xi_{3}\right)$ & $\mathrm{Z} *_{3}\left(\xi_{2}\right)$ & $\mathrm{X} *_{3}\left(\xi_{2}\right)$ & \\
\hline 22 & 0 & 0 & 0 & 0 & 0 & \\
\hline 23 & 100 & 15 & 100 & 15 & 0 & \\
\hline 24 & 200 & 21 & 200 & 21 & 0 & \\
\hline 25 & 300 & 44 & 300 & 45 & 300 & \\
\hline 26 & 400 & 44 & 400 & 60 & 300 & \\
\hline 27 & 500 & 50 & 500 & 66 & 300 & \\
\hline
\end{tabular}

Рис. 3. Последовательное отображение результатов вычислений этапа условной оптимизации

\subsection{2. Последовательное отображение найденного решения}

Для отображения результатов этапа безусловной оптимизации и решения задачи нужно нажать кнопку с текстом «Показать оптимальные значения» в файле пособия. Кнопка становится доступной после того, как были просмотрены расчеты условной оптимизации. После нажатия кнопки происходит выделение цветом ячеек Н27 и I27, содержащих соответственно максимальное значение дохода $Z$ * и оптимальное значение параметра $x_{1}^{*}$, затем последовательное по формулам безусловной оптимизации определяются оптимальные значения $x_{2}^{*}, x_{3}^{*}, x_{4}^{*}$, и ячейки с этими значениями выделяются цветом с интервалом в 1,5c (рис. 4). 


\begin{tabular}{|c|c|c|c|c|c|c|c|c|c|}
\hline & $\mathrm{A}$ & $\mathrm{B}$ & $\mathrm{C}$ & $\mathrm{D}$ & $\mathrm{E}$ & $\mathrm{F}$ & $\mathrm{G}$ & $\mathrm{H}$ & $\mathrm{I}$ \\
\hline & $\ldots$ & \multicolumn{9}{|c|}{} & & & \\
\hline 21 & $\xi_{\mathrm{k}-1}$ & $\mathrm{Z}^{*}{ }_{4}\left(\xi_{3}\right)$ & $\mathrm{x}_{4}\left(\xi_{3}\right)$ & $\mathrm{Z}^{*}{ }_{3}\left(\xi_{2}\right)$ & $\mathrm{x}^{*}{ }_{3}\left(\xi_{2}\right)$ & $\mathrm{Z}^{*}{ }_{2}\left(\xi_{1}\right)$ & $\mathrm{x}_{2}\left(\xi_{1}\right)$ & $\mathrm{Z}^{*}{ }_{1}\left(\xi_{0}\right)$ & $\mathrm{x}^{*}{ }_{1}\left(\xi_{0}\right)$ \\
\hline 22 & 0 & 0 & 0 & 0 & 0 & 0 & 0 & & \\
\hline 23 & 100 & 15 & 100 & 15 & 0 & 15 & 0 & & \\
\hline 24 & 200 & 21 & 200 & 21 & 0 & 24 & 100 & & \\
\hline 25 & 300 & 44 & 300 & 45 & 300 & 45 & 0 & & \\
\hline 26 & 400 & 44 & 400 & 60 & 300 & 60 & 0 & & \\
\hline 27 & 500 & 50 & 500 & 66 & 300 & 69 & 100 & 69 & 0 \\
\hline
\end{tabular}

Рис. 4. Последовательный показ оптимальных значений

В данной задаче максимальное значение дохода равно 69, при этом в первое предприятие нужно вложить средства в размере 0 ден. ед., во второе - 100, в третье 300 и в четвертое - 100. При просмотре результатов сначала происходит заливка ячеек H27 и I27, затем через 1,5c - заливка ячейки G27, затем через 1,5c - ячейки E26, и потом ячейки С23.

\section{3. ВЫВОДЫ И ПЕРСПЕКТИВЫ ДАЛЬНЕЙШИХ ИССЛЕДОВАНИЙ}

Описанный в статье электронный ресурс, предназначенный для изучения метода динамического программирования на примере решения задачи об оптимальном распределении ресурсов, представляет собой разработанный программный продукт, который успешно используется в преподавательской практике. Результаты применения показали его эффективность для усвоения студентами многошагового метода решения задач оптимизации и приобретения практических навыков использования динамического программирования при принятии решений. После изучения теоретического материала примерно 55\% пользователей пособия, которым необходимо решить задачу о вложении средств на практических занятиях, находят правильное решение с первого раза, о чем свидетельствуют результаты автоматической проверки. Примерно 20\% обучающихся определяют нужное решение со второго раза. После многократных проверок к правильному решению самостоятельно приходят 10\% обучающихся, и $15 \%$ пользователей просматривают процесс решения в силу того, что не могут самостоятельно решить задачу. Эффективность данного учебного ресурса проявляется при его использовании для самостоятельного изучения. Многие пользователи осуществляют просмотр решения задачи для самопроверки усвоенного материала, а также для ознакомления с возможностями компьютерных технологий по созданию анимационных эффектов. После самостоятельной работы с пособием на практических занятиях примерно $82 \%$ пользователей решают поставленную задачу с первого раза, при этом задача решается с новыми данными, т.к. при описанной технологии работы пособия полностью одинаковые формулировки задач исключены. Это объясняется более глубоким усвоением материала при многократном решении и просмотре процесса решения задач.

Электронное пособие может быть использовано в рамках таких дисциплин, как «Теория принятия решений», «Методы оптимальных решений» и т.п. Пособие можно модифицировать с учетом специфики преподавания благодаря тому, что оно создано с помощью широко известной и доступной программы Microsoft Excel, при его разработке используются классические приемы программирования.

В пособии можно предусмотреть различные варианты автоматической проверки правильности решения задачи. Например, при необходимости можно организовать проверку на отдельном листе, чтобы еe результаты были доступны только 
преподавателю. Задача об оптимальном распределении ресурсов может иметь несколько решений, т.е. может возникнуть такая ситуация, что получить максимальный доход можно при нескольких вариантах значений переменных $x_{1}^{*}, x_{2}^{*}, x_{3}^{*}, x_{4}^{*}$. В этом случае ориентироваться нужно в первую очередь на оптимальное значение целевой функции (максимальный доход), оно в любом случае остается одним и тем же для всех наборов $x_{1}^{*}, x_{2}^{*}, x_{3}^{*}, x_{4}^{*}$. Для усовершенствования электронного ресурса нужно предусмотреть нахождение и хранение всех вариантов решения задачи, если их несколько, для того, чтобы при проверке подтверждалась правильность любого решения. В разработке, описанной в статье, определяется одно решение, но в этом случае все программные алгоритмы являются негромоздкими и читаемыми.

\section{СПИСОК ЛИТЕРАТУРЫ}

[1] Д. С. Антонюк, “Программно-иммитационные комплексы для формирования экономических компетентностей студентов технических специальностей: критерии и показатели отбора”, Информационные технологии и средства обучения, 2018, Т. 64, № 2, С. 73-87.

[2] Р. В. Манн, "Некоторые аспекты использования информационно-коммуникационных технологий в подготовке будущих экономистов”, Информационные технологии и средства обучения, 2018, Т. 64, № 2, С. 170-184.

[3] О. В. Дубинина, Л. Д. Грицяк, “Использование информационных технологий в обучении будущих проект-менеджеров”, Информационные технологии и средства обучения, 2018, Т. 63, № 1, С. 107 115 .

[4] О. И. Тютюнник. "Методические аспекты проведения лабораторных работ в будущих менеджеров-экономистов средствами системы MAPLE” Современные информационные технологии и инновационные методики обучения в подготовке специалистов: методология, теория, опыт, проблемы: сб. науч. тр. Выпуск 31. Редкол.: И.А. Зязюн и др., 2012, Киев-Винница: ООО Фирма "Планер", С. 482-484.

[5] И. В. Андрощук, И. П. Андрощук. "Технология разработки электронного пособия по дисциплине «Методика трудового обучения»”, Информационные технологии и средства обучения, 2017, Т. 61, № 5, C. 24-35.

[6] В. М. Михалевич, Я. В. Крупський, Ю. В. Добранюк. "Разработка электронных образовательных ресурсов в среде СКМ Марlе", Математика и информатика в высшей школе: вызовы современности: сб. науч. трудов по материалам Всеукр. науч.-практ. конф., 18-19 мая 2017 г.

[7] В. М. Михалевич, О. И. Тютюнник. "Проектирование учебных задач по линейному программированию с использованием систем компьютерной математики”, Информационные технологии и средства обучения, 2013, Т. 38, № 6, С. 123-137.

[8] А. А. Федосеев, “Электронные образовательные ресурсы: эффективность применения”, Cистемы и средства информатики, 2012, Т. 22, № 2, С. 263-277.

[9] Я. В. Крупский, “Проверка эффективности использования учебных Марlе-тренажеров для организации самостоятельной работы студентов”, Информащионные технологии и средства обучения, 2012, Т. 27, № 1, С. 13-25.

[10] Н. В. Бахмат, Т. Ю. Дудка, В. В. Любарец. “Использование мультимедийных образовательных технологий как условие качественной подготовки менеджеров социокультурной деятельности”, Информационные технологии и средства обучения, 2018, Т. 64, № 2, С. 98-109.

[11] Е. В. Архангельская, “Об одном программном средстве мультимедийной поддержки учебных курсов по информатике”, Прикладная информатика, 2017, Т. 12, № 4 (70), С. 74-80.

[12] В. Е. Величко. “Создание электронных образовательных курсов средствами свободного программного обеспечения”, Информационные технологии и средства обучения, 2017, Т 60, № 4, C. $128-140$.

[13] А. А. Панюкова, Т. А. Макаровских, В. И. Дударева, “Обучение компьютерной графике с использованием свободно распространяемого программного обеспечения”, Прикладная информатика, 2014, № 6 (54), С. 37-46.

[14] Е. В. Архангельская, “Об одной реализации метода динамического программирования для решения задачи о замене оборудования с помощью прикладной программы”, Системы и средства информатики, 2018, Т. 28, № 2, С. 178-188. 


\title{
МОЖЛИВОСТІ ВИКОРИСТАННЯ МІСRОSОFТ ЕХСЕL ДЛЯ РОЗРОБКИ ПРОГРАМНИХ ЗАСОБІВ РОЗВ'ЯЗАННЯ ЗАДАЧ ДИНАМІЧНОГО ПРОГРАМУВАННЯ
}

\author{
Архангельська Катерина Владиславівна \\ кандидат фізико-математичних наук, доцент, доцент \\ Саратовська державна юридична академія, м. Саратов, Росія \\ ORCID ID 0000-0003-4108-9876 \\ katysar@rambler.ru
}

\begin{abstract}
Анотація. У статті розглянуто процес створення і розробки електронного посібника для вивчення методу динамічного програмування. Даний метод використовується для вирішення деяких задач оптимізації і вивчається в рамках таких дисциплін, як «Теорія прийняття рішення» і «Методи оптимальних рішень». У статті висвітлено можливості використання розробленого посібника для формування умови і розв'язування практичної задачі про оптимальний розподіл ресурсів між чотирма підприємствами, визначенні максимального доходу і розмірів коштів, що вкладаються в кожне підприємство. Показано, яким чином можна забезпечити унікальність варіантів даної задачі на кожному комп'ютері. Продемонстровано реалізацію автоматичної перевірки правильності відповіді, введеної користувачем, і показ розрахунків, одержуваних на різних етапах виконання завдання, у мультимедійному режимі. При постановці задачі про розподіл ресурсів та проведення розрахунків методом динамічного програмування вихідні дані та результати розрахунків записуються у вигляді таблиць. 3 цієї причини в якості програмного продукту для розробки посібника був обраний широко розповсюджений табличний процесор Microsoft Excel. Для функціонування програмної розробки застосовується програмування в середовищі VBA Excel. Формування умови завдання здійснюється за допомогою програмного коду, що виконується при відкритті файлу електронного посібника. У разі, якщо в користувача виникають труднощі при вирішенні завдання, він може переглянути процес розв'язку в мультимедійному режимі. У статті зазначено, як можна організувати показ розв'язку при натисканні кнопок, розташованих на аркуші Excel. Електронний посібник є цілісним програмним продуктом, який успішно використовується на практичних заняттях у комп'ютерних аудиторіях. Посібник може використовуватися для самостійного i дистанційного навчання.
\end{abstract}

Ключові слова: електронні навчальні ресурси; електронний посібник; динамічне програмування; задача про оптимальний розподіл ресурсів; автоматична перевірка розв'язку; мультимедійність.

\section{POSSIBILITIES OF USING MICROSOFT EXCEL FOR DEVELOPMENT OF SOFTWARE TOOLS FOR SOLVING DYNAMIC PROGRAMMING PROBLEMS}

\author{
Ekaterina V. Arkhangelskaya \\ PhD in Physics \& Mathematics, Associate Professor \\ Saratov State Law Academy, Saratov, Russian Federation \\ ORCID ID 0000-0003-4108-9876 \\ katysar@rambler.ru
}

\begin{abstract}
The article describes the process of creating and developing an electronic textbook for studying the method of dynamic programming. This method is used to solve some optimization problems and is studied in such disciplines as "Theory of decision-making" and "Methods of optimal solutions". Possibilities of use of the developed grant for forming of a condition and a solution of a practical task of the optimal allocation of resources between the four companies, determining the maximum income and the amount of funds invested in each company are covered in article. The article shows how to ensure the uniqueness of variants of the considered problem on each computer. The implementation of automatic verification of the answer entered by the user and display of calculations obtained at different stages of solving the problem in multimedia mode is demonstrated. At problem definition about distribution of resources and carrying out
\end{abstract}


calculations by method of a dynamic programming basic data and calculation results are presented in the form of tables. For this reason, a widely used table editor Microsoft Excel was chosen as a software product for the development of the manual. Programming in the environment of VBA Excel is applied to work of the textbook. The formation of the conditions of the task is carried out using the program code that is executed when you open the file of the electronic manual. If the user has difficulty in solving the problem, he can view the solution process in multimedia mode. The organization of the solution display occurs when you click the buttons located on the Excel sheet. The electronic manual is a complete software product that is successfully used on a practical training in computer classes. The manual can be used for self-study and distance learning.

Keywords: e-learning resources; electronic manual; dynamic programming; the problem of optimal resource allocation; automatic verification of solutions; multimedia.

\section{REFERENCES (TRANSLATED AND TRANSLITERATED)}

[1] D. S. Antoniuk, "Business simulations for the formation of economic competence of technical specialties students: criteria and indicators of selection", Information Technologies and Learning Tools, 2018 , Vol. 64, No 2, p. 73-78 (in Ukrainian).

[2] R. V. Mann, "Some aspects of information and communication technologies application in preparation of future economists", Information Technologies and Learning Tools, 2018, Vol. 64, No 2, p. 170-184 (in Ukrainian).

[3] O. V. Dubinina, L. D. Hrytsiak, "Use of information technologies in future project managers' training”, Information Technologies and Learning Tools, 2018, Vol. 63, No 1, p. 170-115 (in English).

[4] O. I. Tyutyunnik. "Methodical aspects of laboratory work at future managers-economists by means of the MAPLE system" Modern information technologies and innovative teaching methods in the training of specialists: methodology, theory, experience, problems. Vol. 31. Editorial board: I.A. Zyazyun and others. 2012. Kiev Vinnitsa: Firm "Planer", p. 482-484.

[5] I. V. Androshchuk, I. P. Androshchuk, "Technology for development of electronic textbook on handicrafts methodology", Information Technologies and Learning Tools, 2017, Vol. 61, No 5, p. 24-35 (in Ukrainian).

[6] V. M. Mykhalevych, Y. V. Krupskyy, Y. V. Dobranyuk. "Development of electronic educational resources in the SCM Maple environment", Mathematics and Informatics in higher education: challenges of our time: collection of scientific papers on the materials of all-Ukrainian. science.-prakt. Conf., 18-19 May 2017.

[7] V. M. Mykhalevych, O. I. Tyutyunnik. "Design of educational problems on linear programming using systems of computer mathematics". Information Technologies and Learning Tools, 2013, Vol. 38, No 6, p. 123-137 (in Ukrainian).

[8] A. A. Fedoseev, "Electronic educational resources: efficiency of application", Systems and Means of Informatics, 2012, Vol. 22, No 2, p. 263-277 (in Russian).

[9] Y. V. Krupskyy. "Check of the efficiency of educational maple-simulators for organization of students independent work". Information Technologies and Learning Tools, 2012, Vol. 27, No 1, p. 13-25 (in Ukrainian).

[10] N. V. Bakhmat, T. Y. Dudka, V. V. Liubarets, "Multimedia education technologies usage as the condition for quality training of the managers of socio-cultural activity", Information Technologies and Learning Tools, 2018, Vol. 64, No 2, p. 98-109 (in Ukrainian).

[11] E. V. Arkhangelskaya, "Multimedia software development in the educational process", Journal of Applied Informatics, 2017, Vol. 12, No 4 (70), p. 74-80 (in Russian).

[12] V. Y. Velychko, "Creating e-learning means of free software", Information Technologies and Learning Tools, 2017, Vol. 60, No 4, p. 128-140 (in Ukrainian).

[13] A. A. Panyukova, T. A. Makarovskikh, V. I. Dudareva, "On the experience of computer graphics lessons using free software", Journal of Applied Informatics, 2014, No 6 (54), p. 37-46 (in Russian).

[14] E. V. Arkhangelskaya, "About one implementation of the method of dynamic programming to solve the problem of equipment replacement with the application program", Systems and Means of Informatics, 2018, Vol. 28, No 2, p. 178-188 (in Russian).

\section{(c) EY-NC-SA}

This work is licensed under Creative Commons Attribution-NonCommercial-ShareAlike 4.0 International License. 